\title{
Estratégias de enfrentamento (coping) do adolescente com câncer
}

\author{
The coping strategies and the adolescent with cancer
}

\author{
Solange Regina Signori Iamin ${ }^{[a]}$, Ivete Palmira Sanson Zagonel ${ }^{[b]}$
}

[a] Psicóloga, Mestranda do Programa de Pós-Graduação em Biotecnologia em Saúde da Criança e do Adolescente da Faculdades Pequeno Príncipe (FPP), docente, supervisora e consultora em acompanhamento terapêutico, Curitiba, PR - Brasil, e-mail: srsiamin@gmail.com

[b] Enfermeira, docente do Programa de Pós-Graduação em Biotecnologia em Saúde da Criança e do Adolescente da Faculdades Pequeno Príncipe (FPP), diretora acadêmica (FPP), Curitiba, PR - Brasil.

\section{Resumo}

O câncer é uma doença que traz o rótulo de morte antecipada, sendo vista como de difícil recuperação. Quando essa doença afeta o adolescente, emergem duas formas de adaptação: a adaptação à adolescência normal e a adaptação à doença. Para enfrentar o tratamento do câncer, o adolescente utiliza diferentes estratégias de enfrentamento (coping). Conhecer essas estratégias pode ser um caminho para proporcionar melhor qualidade de vida a esses pacientes. O presente estudo tem como objetivos apresentar uma revisão teórica sobre as mudanças que ocorrem no processo de desenvolvimento da adolescência, em seus aspectos biológicos, psicológicos e sociais; mostrar o adoecimento pelo câncer no adolescente e discutir suas estratégias de coping para superação das dificuldades advindas dessa vivência. $\mathrm{O}$ método adotado foi a revisão de literatura mediante a consulta em livros e artigos indexados. A partir da reflexão do conteúdo estudado, percebeu-se que o coping é uma resposta com o objetivo de aumentar, criar ou manter a percepção de controle pessoal.

Palavras-chave: Adolescente. Câncer. Estresse. Adaptação psicológica.

\section{Abstract}

Cancer is a disease that shows a pre-death label, being considered of difficult recovery. When that disease affects the adolescent, it emerges two adjustment ways: the "normal adolescence" adjustment and the disease adjustment. To face the cancer treatment, the adolescent uses different facing strategies (coping). Knowing those strategies can be 
a way to provide better quality of life to those patients. The objectives of the current study is to present a theoretical review about the changes that happen in the adolescent development process, in its biological, psychological and social aspects; to show the coming down with cancer in the adolescent and discuss his/her coping strategies to overcome the difficulties that happen from that experience. The adopted method was the literature review, by consulting books and indexed articles. From the reflection about the studied content, it was noted that coping is an answer with the objective to increase, develop or maintain the personal control perception.

Keywords: Adolescent. Cancer. Stress. Psychological adaptation.

\section{Introdução}

A adolescência é uma época excitante, quando tudo parece possível. Os adolescentes estão no limiar do amor, da vida profissional. Eles estão conhecendo as pessoas mais interessantes do mundo: eles mesmos (Papalia \& Olds, 2000). Essa é uma época em que o jovem pode divertir-se de diferentes maneiras sem ter que assumir responsabilidades e obrigações que o adulto assume, porém, é preciso pensar essa etapa do ciclo vital com um olhar que perceba o período difícil e ao mesmo tempo importante que o adolescente está vivenciando. Esses jovens estão em desenvolvimento interno e externo, tomando decisões que por certo influenciarão toda a sua vida. $\mathrm{Na}$ adolescência, o jovem inicia a busca pela sua independência, decidindo o que quer fazer, as coisas que gosta e os projetos de vida. Começa, também, a olhar para fora da família procurando a convivência e a integração com o grupo social em que está inserido. Tais mudanças geram conflitos necessários ao amadurecimento do indivíduo.

Inclua-se ao panorama ora exposto, a descoberta e a experiência de um câncer, considerada uma das doenças mais perturbadoras no ser humano. Assumir o seu tratamento, por vezes longo e doloroso, é uma tarefa difícil, já que "uma doença duradoura leva a uma fundamental reconsideração da biografia da pessoa e de seu conceito de si” declara Michael Bury, citado por Herzlich (2004, p. 387).

Passar pelos procedimentos e pelos efeitos consequentes das prescrições médicas para cada caso é experimentar sentimentos de estranheza, insegurança, revolta, impotência e, principalmente, de não se reconhecer mais. A vivência é permeada por sentimentos de mutilação que extrapolam as físicas; é uma experiência de perdas e de incerteza acerca do porvir. $\mathrm{O}$ viver com câncer é um viver intenso, é um abrir-se às emoções plenas e, também, um penoso forjar da fragilidade em força. Assim, passar pela experiência de estar com uma doença tão fortemente vinculada à morte é sofrer uma série de interposições no projeto de vida; é ter a vida em suspenso (Silva, 2005a).

Refletir sobre a maneira como o adolescente enfrenta a fase desenvolvimental e a simultaneidade da ocorrência do adoecimento por câncer é possibilitar a contextualização do fato, como uma etapa de mobilizações significativas no seu universo de adolescer/adoecer.

Nesse caminho surgem estressores importantes, como a hospitalização, que não estão relacionados apenas à doença em si, mas carregam muitos fatores que afetam sobremaneira a vida de quem a vivencia. Entre esses fatores inclui-se a dor; o ambiente hospitalar pouco familiar; a exposição a procedimentos médicos invasivos; a separação dos pais; o estresse dos acompanhantes; a ruptura da rotina de vida e adaptação a uma nova rotina imposta e desconhecida; a perda da autonomia, controle e competência pessoal; a incerteza sobre a conduta mais apropriada; e a morte (Méndez, Ortigosa \& Pedroche, 1996).

Tendo em vista essa contextualização, surgem questionamentos sobre como os adolescentes enfrentam as mudanças que vão acontecendo simultaneamente, aquelas que fazem parte do processo de desenvolvimento da fase adolescente (desenvolvimentais) e aquelas próprias do adoecimento (situacionais). Que estratégias o adolescente utiliza para enfrentar essas mudanças e como enfrenta o adoecimento e as adversidades que decorrem desse adoecer? De alguma maneira, é preciso identificar como esse jovem enfrenta o adoecimento pelo câncer no transcorrer da sua dor física e emocional com a doença, para que tal informação possa ser utilizada pelos profissionais da saúde envolvidos em seu cuidado.

Ademais, a compreensão dessa rede de modificações impostas pelo câncer no adolescente 
e das estratégias de enfrentamento utilizadas por ele diante dessa vivência pode favorecer intervenções de apoio mais eficazes, a partir da captura de possíveis alterações, dentro desse processo, que sejam prejudiciais ao viver humano.

Buscando atender às questões expostas neste artigo de revisão, foram estabelecidos os objetivos de contextualizar as mudanças que ocorrem no processo de desenvolvimento da adolescência, sejam biológicas, psicológicas ou sociais; o adoecimento pelo câncer; e discutir as estratégias de coping para a superação das dificuldades advindas dessa vivência.

\section{A adolescência e o processo de desenvolvimento}

Desde o nascimento, a criança passa por um processo de crescimento e amadurecimento físico e psicológico. A entrada na adolescência é marcada por mudanças desse novo viver, é uma etapa de enfrentamento de conflitos internos e externos. Nessa fase, a aparência física se torna uma referência na autoavaliação que o adolescente faz de si mesmo. "O corpo e os traços apresentam importante relação com a imagem que tem de si e com a ideia que faz de como é aos olhos dos outros" (Campos, 1977, p. 23). O jovem mostra-se ligado à sua imagem corporal, olhando-se no espelho, tentando identificar as mudanças que vão acontecendo, manifestando, então, sua aprovação ou desaprovação em relação à imagem que vê refletida.

As mudanças físicas que vão acontecendo nessa etapa mostram as alterações do processo evolutivo, mudança no timbre da voz, aparecimento de pelos no corpo, cravos, espinhas, amadurecimento sexual, mudanças nas feições do rosto, crescimento desordenado de pés e mãos. Ainda não acostumado com seu novo físico, "O adolescente perde a noção do espaço que ocupa, e é por isso que, sem querer, torna-se sem jeito" (Novello, 2004, p. 21).

Para Gottlieb, citado por Patton e Viner (2007), os conceitos atuais de adolescência são tipicamente marcados por importantes modificações biológicas na puberdade e por transições sociais extremamente variáveis. Os processos biológicos têm início na interação da puberdade com o contexto social, para afetar o desenvolvimento emocional e social do indivíduo. A partir dessa interação, decorre a adolescência, como um "derivado da estrutura sócio-econômica” (Bock, 1996, p. 253). Compreende-se, então, que na adolescência, há uma correlação entre as alterações dos aspectos biológicos, físicos, psicológicos e sociais.

Aberastury (1973, p. 18) entende que a adolescência é uma fase de contradições, confusões e ambivalências, assinalada por fricções entre o ambiente familiar e o ambiente que circunda o adolescente. Viver uma adolescência 'normal' traz consigo questionamentos e dúvidas, gerando ansiedade pelo desconhecido mundo que está sendo descoberto e a constante busca de atenção e aprovação. É natural, ao longo desse processo do desenvolvimento biopsicossocial do indivíduo, ocorrerem "situações marcantes que traduzem essa ruptura em novas realidades e percepções sobre a sua existência, consideradas como rituais de passagem da adolescência" (Bretas, 2004, p. 405).

Nessa etapa de desenvolvimento o adolescente vai pouco a pouco descobrindo e construindo sua própria identidade, vai conquistando novos espaços, liberando-se da família, buscando sua autonomia, iniciando a consciência de si mesmo como uma pessoa diferente das demais, que conquista seu próprio grupo de amigos, que consegue definir pensamentos, sentimentos e desejos e que também decide por si próprio. Assim, as tarefas da adolescência começam com "um rápido crescimento físico e maturação sexual durante a puberdade e, com isso, aceleram os movimentos que buscam solidificar uma identidade e estabelecer a autonomia em relação à família" (Carter \& Macgoldrick, 2001, p. 225).

Tantas mudanças indicam um desenvolvimento permeado por questões pelas quais o adolescente passa, desde situações mais corriqueiras sobre como portar-se adequadamente, até situações mais complexas, como a busca de sua própria identidade, que é a tarefa mais importante dessa fase, segundo Erikson (1976). É nesse contexto de conflitos desenvolvimentais que segue a discussão acerca das reações do adolescente diante do diagnóstico e vivência de um câncer.

\section{O adoecimento pelo câncer e o adolescente}

O câncer é uma doença de grande impacto emocional no ser humano (Silva, 2005b) e aquela que mais afeta a imagem corporal do adolescente, pois 
marca visivelmente o corpo em desenvolvimento pela alopecia, perda ou aumento de peso, mal-estar constante causados pelos procedimentos terapêuticos, fazendo com que se esconda em si mesmo. Há a reclusão social, geralmente em seu quarto, em sua casa, preterindo o que poderia ser uma das mais ricas experiências, a de oportunizar o desenvolvimento de potencialidades diante da adversidade.

Em pesquisa sobre câncer na infância e adolescência, realizada no ano de $2004 \mathrm{em}$ hospital de referência no tratamento de câncer em Curitiba, ficou demonstrado que, de um total de 27.987 casos que foram admitidos pelo Registro Hospitalar de Câncer (RHC) no período de janeiro de 1990 a dezembro de 2002, 880 foram de tumores em pacientes menores de 15 anos, representando 3,1\% do total de casos admitidos, em que $40 \%$ eram do sexo feminino e 60\% do sexo masculino; e 377 casos de tumores em pacientes adolescentes entre 15 e 19 anos, representando $1,3 \%$, sendo $46 \%$ do gênero feminino e $53,8 \%$ do gênero masculino (Liga Paranaense de Combate ao Câncer, 2004).

O câncer infanto-juvenil, até 18 anos, é considerado raro quando comparado com os tumores que afetam os adultos. Correspondem a percentuais entre $1 \%$ e $3 \%$ de todos os tumores malignos na maioria das populações. Em geral, a incidência total de tumores malignos na infância é maior no sexo masculino. Do ponto de vista clínico, os tumores pediátricos apresentam menores períodos de latência, em geral crescem rapidamente e são mais invasivos (INCA, 2010).

De acordo com Smith e Ries citados por Rodrigues e Camargo (2003), nos Estados Unidos, em 1998, foram diagnosticados cerca de 12.400 casos novos de câncer entre crianças e adolescentes menores de 20 anos, constituindo a primeira causa de óbito por doença em crianças maiores de um ano. Contudo, ainda é uma doença incomum e o seu diagnóstico é frequentemente retardado.

Ao receber o diagnóstico de câncer, o adolescente é compelido a enfrentar a vivência de uma doença crônica, que coincide com as mudanças próprias da sua idade, gerando o processo de transitar de uma condição saudável para a condição de doença, alterando os projetos que esse adolescente tinha acerca de si mesmo e de seu mundo.

Segundo Torres (1999), o diagnóstico da doença do câncer na adolescência é visto como de difícil recuperação, implicando um conceito de morte antecipada, de sofrimento sem esperança, perda de projetos futuros, interferindo, assim, na autoimagem adequada e na formação da identidade pessoal, levando a uma perda na qualidade de vida.

Alguns adolescentes, a família e os amigos se calam diante do diagnóstico e o tratamento da doença, pois o impacto não lhes permite falar sobre o que está acontecendo. Talvez essa seja a maneira que encontram para lidar momentaneamente com o problema, ou como forma de negação do agravo a ser enfrentado.

O adoecimento pelo câncer é considerado uma situação estressante, que apresenta sintomas que envolvem irritação, medo, sudorese, dor de cabeça, mal-estar gastrointestinal, náuseas, tonturas e, ainda, sintomas psíquicos que se manifestam quando o sujeito enfrenta uma situação que no momento lhe parece insolúvel.

O processo de adoecer na quase totalidade dos casos traz em seu bojo "uma configuração de total falta de sentido para o próprio significado existencial do paciente" conforme afirma AngeramiCamon (2003, p. 79). O autor afirma, ainda, que um diagnóstico de câncer traz aspectos que transcendem a própria patologia, criando sobre sua ocorrência, fatores mitificados que acabam se tornando realidade pela configuração que é dada. O adolescente imagina a evolução, a cura, as quais são construções de seu imaginário.

O adoecimento é visto como aprisionamento à rotina, a um lugar estranho, sem perspectivas de sair aos locais que antes podia frequentar. A rotina do tratamento induz ao acostumar-se a isso, mas também é expressa como incômodo, chato, difícil, pela repetição das sessões de quimioterapia.

A doença, mesmo sendo fato, somente adquire a condição de fato pela maneira que é apreendida pela consciência. E na consciência que determinados diagnósticos ganham significado de irreversibilidade, de transformação em aspectos que envolvem até mesmo a cura. O adolescente tem dificuldades de compreender essa dimensão a qual está passando, não aceita adoecer, não aceita as manifestações da patologia, não aceita a rotina, entra na turbulência emocional.

Atingir o completo bem-estar a que todo ser humano almeja é certamente impossível, pois ele vivencia diferentes demandas durante o desenvolvimento, as quais o levam também à situação de estresse, que lhe produz algum tipo de pensamento ou comportamento disfuncional e a emissão de 
respostas de intenso sofrimento psíquico. É para amenizar esse sofrimento que o indivíduo utiliza estratégias de coping, nas diferentes vivências que surgem no percurso de sua vida.

\section{Estratégias de coping do adolescente com câncer}

Levando em conta o câncer como uma das doenças mais temidas pelo ser humano, carregada pela incerteza da cura, a ideia de muitas hospitalizações, da sintomatologia pós-quimioterapia, da dor física e emocional e até mesmo da morte, entende-se que o vivenciar dessa doença, certamente, é uma fonte de estresse intenso para o paciente, e também para a sua família. Em razão do grande impacto que o diagnóstico do câncer e os respectivos procedimentos médicos hospitalares causam no adolescente, emerge a importância do estudo acerca do enfrentamento do câncer, para compreender as estratégias de coping que ele, adolescente, utiliza diante da doença.

De acordo com o modelo de processamento de estresse e coping proposto por autores consagrados como Lazarus e Folkman (1984), é a partir do evento estressante que surge a ameaça, prejuízo e desafio. É nesse momento que a pessoa pode questionar o que pode fazer, que resultado espera e qual o custo desse empreendimento. Somente com essa reflexão surgem as estratégias de coping focadas no problema (forma ativa e direta de eliminar ou diminuir o problema, por exemplo, obtendo informações sobre o problema, traçar objetivos, entre outros) ou na emoção, fazendo com que ocorra uma alteração das reações diante do problema (pensamento positivo, aceitação, negação) chegando a um resultado de mudança e de sentir-se melhor. Assim, "o coping funciona como um mediador entre o estressor e o resultado advindo desse estressor" (Antoniazzi, Dell'Aglio \& Bandeira, 1998, p. 277).

Apesar de não haver um padrão específico de estratégias de coping, Almanza-Muñoz e Holland (2000) salientam que existem três fases pelas quais os pacientes oncológicos passam: fase de negação, em que o paciente tem um sentimento de anestesia emocional, assim como uma descrença de que esse diagnóstico seja verdadeiro; um período de confusão, em que há certa agitação emocional na qual o paciente vai aceitando, aos poucos, a realidade da doença e, em uma fase mais longa, o paciente começa a voltar à sua rotina, época em que ele vai tendo esperança quanto ao tratamento.

Ainda, o coping é conceituado como "uma resposta com o objetivo de aumentar, criar ou manter a percepção de controle pessoal" (Savóia, 1999, p. 6). As pessoas são consistentes na maneira como percebem e combatem o estresse, porém, essa consistência vai depender das variações de situações e de estilo, em que cada um escolhe uma determinada estratégia, dependendo do seu repertório individual e das experiências que foram reforçadas até então.

A psicologia, a enfermagem e outras profissões da área de saúde ou humanas têm estudado e pesquisado o conceito de coping como sendo uma maneira do indivíduo demonstrar sua forma de adaptação às situações estressantes vivenciadas no seu dia a dia. O conceito de coping parece ter hoje uma definição importante para pensar o enfrentamento do estresse nas diferentes situações pelas quais o indivíduo passa.

Utilizar estratégias de coping auxilia a buscar o ajustamento positivo diante do estressor, é uma resposta envolvendo uma reação emocional ou comportamental espontânea, orientada para a redução do estresse. A disponibilidade de recursos afeta a avaliação do evento ou situação, os quais envolvem as características da pessoa, as características do estressor e as características do contexto, todos interligados.

O estresse é "a relação que se estabelece entre as situações ou acontecimentos perturbadores e as reações (sentimentos, pensamentos e comportamentos) do organismo" (Costa \& Leal, 2006). O coping, por sua vez, especifica o comportamento do organismo diante de fatores estressantes. Independentemente de como se conceituam os processos de avaliação da situação estressante e coping, o importante é que ambos influenciam na adaptação do sujeito, tendo em vista que o objetivo é diminuir a probabilidade da situação estressante causar algum prejuízo e/ou redução das reações emocionais negativas.

Embora a prevalência e severidade de distúrbios psicosociais em crianças e adolescentes com câncer sejam controversas, o importante papel moderador de coping é amplamente aceito como auxiliar no funcionamento psicossocial desse grupo (Sanger etal. citados por Hampel, Rudolph, Stachow, Laß-Lentzsch \& Petermann, 2005). 
O coping pode mediar a relação entre as situações desafiadoras e o seu impacto nos resultados psicossociais. Os sobreviventes de câncer, em longo prazo, continuam enfrentando uma série de desafios em sua vida diária, e para que tenham um enfrentamento positivo precisam desenvolver uma rede social de apoio, caso contrário, conforme mostra o estudo de Zucca et al. (2010), poderão desenvolver respostas "desadaptativas" de enfrentamento.

Em estudo sobre câncer infantil, foi elaborado um instrumento de avaliação das estratégias de enfrentamento à hospitalização, em que os resultados indicaram um padrão de respostas de enfrentamento mais facilitador (brincar, conversar, tomar remédio e rezar) do que não facilitador (esconder-se, brigar, sentir culpa, fazer chantagem). O coping como perspectiva comportamental-cognitiva foi adotado nesse estudo (Motta \& Enumo, 2004).

Chapman e Pepler (1998) em estudo sobre as estratégias de coping, relacionado à esperança e sofrimento antecipatórios em famílias com membros em cuidados paliativos, encontraram que $\mathrm{o}$ enfrentamento e a esperança são dois fatores que podem influenciar o sofrimento antecipatório, e que o enfrentamento tem sido visto tanto como um traço quanto como um processo. Tais autores referem que alguns teóricos veem uma situação específica como um processo de enfrentamento, mas que não negam a existência de um estilo geral de enfrentamento e que esse pode influenciar a escolha de estratégias de enfrentamento em dada situação.

Arbizu (2000) acentua que se deve permitir ao paciente responder com as suas próprias estratégias de enfrentamento, de adaptação, aquelas coerentes ao seu próprio estilo de personalidade e sem a repressão das emoções que possam surgir/sentir.

Anarte et al. (2001), em estudo feito com pacientes crônicos, da chamada personalidade C, aquela cuja variável de personalidade é mediadora do processamento emocional, modulando o processamento da informação que o indivíduo realiza diante da doença crônica, entendida como experiência estressante, encontrou que esses pacientes utilizam determinadas estratégias de enfrentamento como controle emocional, repressão emocional, necessidade de harmonia, compreensão e racionalização.

Bárez, Blasco e Castro (2003) relatam em sua pesquisa o juízo de controle em pacientes com câncer, que no caso dessa doença a sensação de controle pode acontecer quando o paciente acredita que o médico, por meio dos tratamentos de quimioterapia, pode conseguir a cura.

Hampel et al. (2005) realizaram um estudo sobre enfrentamento entre crianças e adolescentes com doença crônica. O estudo objetivou investigar os efeitos da idade e gênero sobre o enfrentamento com estressores diários entre crianças e adolescentes com doença crônica e controles saudáveis, combinados por idade, gênero e grau escolar. Foram coletados dados sobre enfrentamento a estressores acadêmicos e interpessoais. Os resultados indicaram que o enfrentamento com estressores diários foi melhorado em crianças e adolescentes com doença crônica, comparado aos controles saudáveis. Assim, os pacientes demonstraram mais enfrentamento específico de situação de estressores relacionados ao fator social e à escola do que os controles saudáveis. Os resultados sugerem que o enfrentamento de doença crônica pode levar a um enfrentamento mais efetivo de estressores diários.

Kyritsi et al. (2007) estudaram o autoconceito de crianças e adolescentes com câncer. Nesse estudo participaram 165 crianças com câncer, que foram comparadas a 417 crianças saudáveis e 212 crianças com talassemia de idade correspondente. As crianças do estudo e os controles com talassemia foram obtidos de crianças hospitalizadas em dois dos maiores hospitais pediátricos de Atenas: Pan e Agl. Kyriakou e Agia - Sophia. Os controles saudáveis vieram de escolas de Atenas. A coleta de informações foi realizada por meio de entrevista entre o pesquisador e crianças dos três grupos. Para todos os grupos de crianças foi utilizada como instrumento a escala Piers Harris 'Como eu me sinto'. Os resultados da análise de dados demonstraram que crianças com câncer têm uma imagem negativa de si mesmas em comparação aos controles. Em uma análise posterior, elas demonstraram problemas comportamentais, especialmente crianças com menos de 12 anos. Sua doença prejudica seu correto desenvolvimento intelectual e acadêmico e elas se sentem menos felizes e menos satisfeitas do que crianças saudáveis da mesma idade. Concluiu-se que a doença e seu tratamento afetam negativamente o autoconceito de crianças e, de acordo com os resultados, as crianças com câncer avaliam negativamente seu comportamento, aparência, desempenho escolar e expressam menos satisfação e felicidade. 
Till (2004), em pesquisa sobre as estratégias de enfrentamento utilizadas pelos adolescentes na sua experiência com câncer, identificou que eles lidaram com a doença por meio da negação, ou seja, não pensar a respeito do que estava acontecendo com eles e para isso realizavam diversas atividades como ferramentas de distração; manter-se informados a respeito da doença, perguntando aos médicos sobre os procedimentos a serem realizados e dando continuidade aos estudos por meio de programas que incluíam o acompanhamento dos adolescentes e de suas atividades escolares por professores que iam até o hospital ou suas casas, e os grupos de apoio, os quais possibilitaram a percepção de estarem fazendo novos amigos, com os quais se sentiam identificados.

Silva e Sá (2010) explicitam que a música, utilizada como terapia, constitui-se em um importante canal de comunicação e expressão das perdas, ativando o potencial de vida do próprio ser. A música deu voz ao silêncio, ao sofrimento, aos sentimentos profundos, encorajando o adolescente a lidar com as dificuldades advindas da doença e do próprio tratamento, auxiliando-os no desenvolvimento de estratégias de enfrentamento ao estresse.

Um estudo sobre coping realizado por Kyngäs, Mikkonen, Nousiainen, Rytilahti, Seppänen e Vaattovaara et al. (2001), citados por Maia e Correia (2008), com pacientes oncológicos comidades entre 16 e 22 anos, apontou que as estratégias de coping utilizadas pelos adolescentes foram o suporte social, principalmente a família, a crença em uma rápida recuperação e retorno à vida normal, além de buscar informações sobre a doença.

Sorgen e Manne (2002), citados por Maia e Correia (2008), pesquisaram crianças e adolescentes com câncer e encontraram que o uso das estratégias de coping focadas no problema estavam relacionadas à percepção de maior controle da doença, enquanto as estratégias focadas na emoção estavam associadas à percepção de níveis mais baixos de controle.

O estresse é uma reação emocional e física a uma mudança, a qual suscita a utilização de diferentes estratégias de coping pelas pessoas. É possível lidar com o estresse observando alguns sinais, os quais, de acordo com a época de aparecimento, duração e consequências, podem ser prevenidos ou evitados, conhecendo a causa ou mesmo mudando a maneira de reagir a determinados problemas. Dentre as estratégias que a pessoa pode usar, encontram-se: fazer atividades que promovam relaxamento como ioga, respiração, meditação, exercícios; praticar um bobby; aprender a aceitar as coisas que não podem ser modificadas; pensar positivamente; estabelecer limites; aprender a dizer não; fazer uma coisa de cada vez; dormir oito horas por noite; conversar com a família e amigos sobre os problemas; não enfrentar o estresse de maneira prejudicial à saúde, como comer demais, usar álcool, tabaco ou drogas; procurar ajuda profissional se necessário.

Vários estudos também demonstramaimportância da espiritualidade no enfrentamento de situações estressantes como a doença. Pargament citado por Paiva (2007) refere que as pessoas, quando se deparam com doenças físicas ou mentais, buscam recursos religiosos como oração, promessas, peregrinação, rituais para poder enfrentar tais situações. $\mathrm{O}$ enfrentamento religioso torna-se então um meio de encontrar sentido, estabilidade e conforto diante da doença.

A partir da ativação de estratégias pessoais de coping pelo adolescente, cria-se a possibilidade de crescimento de uma multiplicidade de modos de ser e relacionar-se, possibilidade ampla de construir uma relação com a vida, lutar contra o instituído "O câncer é a morte" e criar um novo lugar para o viver, promover possibilidades de falar e desenvolver novas habilidades sociais, utilizar o ambiente hospitalar ambulatorial como contexto social, conjugar o cuidado com ações na construção de novos projetos de vida.

Qualquer tentativa de administrar o estressor é considerada coping, tenha ela sucesso ou não no resultado, de acordo com o modelo de Lazarus e Folkman (1984).

\section{Consideraçóes finais}

Diante dos argumentos expostos, é possível apreender que as estratégias de enfrentamento (coping) utilizadas pelo adolescente, diante do diagnóstico do câncer, estão relacionadas ao fato de concentrar esforços para solucionar o problema do câncer, pedir ajuda e conselhos aos familiares, ter autocontrole procurando manter-se calmo diante da situação, buscando estratégias de distração, conversar com outras pessoas, tentar esquecer o problema, pensar em coisas que o façam sentir-se melhor.

Essas estratégias de enfrentamento (coping) utilizadas pelos adolescentes possibilitam uma nova 
construção, uma nova experiência, diminuindo os fatores estressantes e promovendo uma reorganização do cotidiano, dando um novo significado à experiência traumática da vivência do câncer.

A literatura aponta que pacientes oncológicos utilizam como estratégias de coping: o suporte social, o foco em aspectos positivos, o distanciamento e o isolamento do problema e evitar pensamentos sobre o câncer, conforme referem Fawzy, Fawzy e Canada (2000) citados por Maia e Correia (2008).

Certamente, no momento em que recebe o diagnóstico de câncer surge a ansiedade, o medo da doença desconhecida e as indagações sobre os diferentes procedimentos que acontecerão a partir de então. $\mathrm{O}$ tratamento exige uma adaptação ao novo espaço pelo qual o adolescente irá circular, no espaço hospitalar, espaço social diferenciado, no espaço dos procedimentos em que a doença, o sofrimento psíquico, a dor corporal e a morte são constantes, impondo uma adaptação a todas as transformações que acompanharão esse tratamento.

Não será diferente o processo de recuperação, pois esse também será um momento difícil, em que o adolescente terá que enfrentar os efeitos causados pelo tratamento medicamentoso, além de enfrentar a sua própria imagem, sua própria tristeza e sua própria luta pela vida, marcados pelas incertezas.

Cabe refletir sobre a importância do profissional de saúde possibilitar, em seu vínculo com o adolescente, ações como compartilhar, escutar, observar, ajudar a desenvolver atividades, estimular, cuidar, aliviar a ansiedade e a angústia, promover a expressão de emoções e sentimentos, podendo assim colaborar para que esse adolescente desenvolva respostas adaptativas à situação da vivência do câncer.

Espera-se que este trabalho de revisão sobre as estratégias de enfrentamento (coping) e o câncer na adolescência possa colaborar no entendimento das consequências de uma doença tão devastadora e, além disso, oferecer subsídios que possam colaborar na recuperação do adolescente portador do câncer, a partir do entendimento da maneira como ele enfrenta essa situação. É fundamental que a área da saúde prossiga pesquisando sobre o coping e a vivência do câncer pelo adolescente, para que se possa promover, com esse conhecimento, a participação de um cuidado humanizado.

\section{Referências}

Aberastury A. et al. (1973). Adolescência. Buenos Aires: Kargieman.

Almanza-Muñoz J. J., \& Holland, J. C. (2000). Psicooncologia: Estado actual y perspectivas futuras. Revista del Instituto Nacional de Cancerología, 46(3), 196-206.

Anarte, M.T.et al. (2001). Evaluación del patrón de conducta tipo $\mathrm{C}$ em pacientes crônicos. Anales de Psicologia, 16(2),133-141. Recuperado em 10 jan. 2010, em http:/ / redalyc.uaemex.mx/pdf/167/16716203.pdf

Angerami-Camon, V.A. (2003). Temas existenciais em psicoterapia. São Paulo: Thomson Learning.

Antoniazzi, A. S., Dell' Aglio D. D., \& Bandeira, D. R. (1998). O conceito de coping: Uma revisão teórica. Estudos de Psicologia, 3(2), 273-294.

Arbizu, J. P. (2000). Factores psicológicos que intervienen en el desarrollo del câncer y en la respuesta de tratamiento. Anales Sis San Navarra, 24(1), 173178. Recuperado em 12 jan. 2010, em http://www. cfnavarra.es/salud/anales/textos/vol24/suple1/ suple16a.html

Bárez, M., Blasco, T., \& Castro, J. F. (2003). La inducción de sensación de control como elemento fundamental de la eficácia de las terapias psicológicas em pacientes com câncer. Anales de Psicologia, 19(2), 235-246. Recuperado em 12 dez. 2009, em http:/ / www.um.es/ analesps/v19/v19_2/06-19_2.pdf

Bock, A. M. B., Furtado, O., Teixeira, M. L. (1996). Adolescência: tornar-se jovem. In A. M. B. Bock, O. Furtado, M. L. Teixeira (Org.). Psicologias: Uma introdução ao estudo de Psicologia (pp. 202-219). São Paulo: Saraiva.

Brêtas, J. R. S. (2004). A mudança corporal na adolescência: A grande metamorfose. Temas sobre Desenvolvimento, 12(72), 29-38.

Campos, D. M. S. (1977). Psicologia da adolescência: Normalidade e psicopatologia. Petrópolis: Vozes.

Carter, B. MackGoldrick, M. (2001). As mudanças no ciclo de vida familiar: Uma estrutura para a terapia familiar. (2a ed). Porto Alegre: Artmed.

Chapman, K., \& Pepler, C. (1998). Coping, hope, and antecipatory grief in family members in palliative home care. Cancer Nursing, 21(4), 226-234. 
Costa, E. S., \& Leal, I. P. (2006). Estratégias de coping em estudantes do ensino superior. Psicológica, 24(2), 189-199. Recuperado em 2 dez. 2008, em http:// www.scielo.oces.mctes.pt/scielo.php?script $=$ sci_ arttext\&pid $=$ S0870-82312006000200006\&lng $=$ pt\& $\mathrm{nrm}=$ iso

Erikson, E. H. (1976). Identidade, juventude e crise. Rio de Janeiro: Zahar.

Hampel, P., Rudolph, H., Stachow, R., Laß-Lentzsch, A., Petermann, F. (2005). Coping among children and adolescent with chronic illness. Anxiety Stress Coping, 18(2), 145-155.

Herzlich, C. (2004). Saúde e doença no início do século XXI: Entre a experiência privada e a esfera Pública. Phisys: Revista Saúde Coletiva, 14(2), 383-394.

Instituto Nacional do Câncer-INCA. (2010). Incidência do câncer infantil. Rio de Janeiro. Recuperado em 18 jan. 2010, em http://www.inca.gov.br/releases/ press_release_view.asp?ID $=1974$

Kyritsi, H. et al. (2007). Self concept of children and adolescents with câncer. Health Science Journal. Recuperado em 15 jan. 2010, em http://www.hsj.gr

Lazarus, R. S., \& Folkman, S. (1984). Stress, appraisal, and coping. New York: Springer.

Liga Paranaense de Combate ao Câncer. (2004). Câncer na infância e na adolescência. Curitiba: LPCC.

Maia, L., \& Correia, C. (2008). Consequências psicológicas, estratégias de coping e intervenção na doença oncológica: Uma revisão da literatura para a aplicação prática. Recuperado em 10 abr. 2009, em www.psicologia.com.pt

Méndez, F. X., Ortigosa, J. M., \& Pedroche, S. (1996). Preparación a la hospitalización infantil (I): Afrontamiento del estrés. Psicologia Conductual, 4(2), 193-209.

Motta, A. B., Enumo, S. R. F. (2004). Câncer infantil: Uma proposta de avaliação das estratégias de enfrentamento da hospitalização. Estudos de Psicologia, 21(3), 193-202.

Novello, F. P. (2004). Psicologia da adolescência: O despertar para a vida. (6a ed.). São Paulo: Paulinas.

Paiva, G. J. (2007). Religião, enfrentamento e cura: Perspectivas psicológicas. Estudos de Psicologia, 24(1), 99-104.
Papalia, D. E., \& Olds, S. W. (2000). Desenvolvimento Humano. (Daniel Bueno Trad). (7a ed.). Porto Alegre: Artes Médicas.

Patton, G. C., \& Viner, R. (2007). Pubertal transitions in health. Murdoch. Childrens Research Institute, University of Melbourne, Australia. Lancet, (369), 1130-1139.

Rodrigues, K. E., \& Camargo B. (2003). Diagnóstico precoce do câncer infantil: responsabilidade de todos. Revista da Associação Médica Brasileira, 49(1), 29-34.

Savoia, M. G. (1999). Escalas de eventos vitais e de estratégias de enfrentamento (coping). Revista de Psiquiatria Clínica, 26(2), 57-67.

Silva, L. C. (2005a). Vozes que contam a experiência de viver com câncer. Psicologia Hospitalar, 3(1), 1-17.

Silva, V. C. E. (2005b). O impacto da revelação do diagnóstico de câncer na percepção do paciente. Dissertação de Mestrado, Universidade de São Paulo. Programa interinstitucional. USP/UEL/UNOPAR. Ribeirão Preto, São Paulo.

Silva, F. O., \& Sá, L. C. Perdas na adolescência: Música como expressão de sofrimento. Recuperado em 27 maio 2010, em http://www.anppom.com.br/anais/ anaiscongresso_anppom_2007/musicoterapia/musicoterap_FOrtins_LCSa.pdf

Till, T. (2004). Coping with cancer: The adolescent experience. Master of Science, School of Nursing, Faculty of Health Science, Austrália.

Torres, W. C. (1999). A criança diante da morte: Desafios. São Paulo: Casa do Psicólogo.

Zucca, A. C. et al. (2010). Life is precious and I'm making the best of it: Coping strategies of long-term cancer survivors. Psycho-Oncology, 19(2), 1268-1276. doi: 10.1002/pon.1686.

Recebido: 09/03/2010

Received: 03/09/2010

Aprovado: 19/05/2010

Approved: 05/19/2010 